\title{
Strain in Nanoscale Germanium Hut Clusters on Si(001) Studied by X-Ray Diffraction
}

\author{
A. J. Steinfort, P. M. L. O. Scholte, A. Ettema, and F. Tuinstra \\ Department of Applied Physics, Delft University of Technology, \\ P.O. Box 5046, NL-2600 G A Delft, The Netherlands \\ M. Nielsen, E. Landemark, D.-M. Smilgies, and R. Feidenhans'1 \\ Risф National Laboratory, DK-4000 Roskilde, Denmark \\ G. Falkenberg, L. Seehofer, and R. L. Johnson \\ II. Institut für Experimentalphysik, Universität Hamburg, Luruper Chaussee 149, D-22761 Hamburg, Germany
}

(Received 28 May 1996)

\begin{abstract}
Scanning tunneling microscopy and synchrotron $\mathrm{x}$-ray diffraction have been used to investigate nanoscale Ge hut clusters on $\mathrm{Si}(001)$. We have been able to identify the contributions to the scattered $\mathrm{x}$-ray intensity which arise solely from the hut clusters and have shown that x-ray diffraction can be very sensitive to the strain field in the hut clusters. At the Ge/Si interface the Ge clusters are almost fully strained with a misfit of only $0.5 \%$ but towards the apex of the clusters the strain is relaxed and the atomic spacing is close to the natural Ge lattice spacing with a 4.2\% misfit. [S0031-9007(96)01009-5]

PACS numbers: 68.35.Bs, 61.10.-i, 61.16.Ch, 68.55.Jk
\end{abstract}

Electronic devices and many other technologies exploit the properties of thin films of various materials with different lattice constants deposited on top of each other. Currently there is great interest in strained-layer superlattices made of III-V semiconductors for laser applications and in SiGe alloys because of their excellent high frequency performance. The internal strain is a very important parameter in these systems because it modifies the optical and electronic properties of the materials. In this paper we address the problem of determining the strain in $\mathrm{Ge}$ hut clusters grown on $\mathrm{Si}(001)$ substrates.

It is conventional to distinguish between three classical growth modes: layer by layer growth (Frank-van der Merwe mode), 3D island growth (Volmer-Weber mode), and layer by layer followed by 3D island growth [StranskiKrastanov (SK) mode] [1]. These are simplified scenarios, and in practice the situation may be complicated by such effects as surface alloying and internal strain that can lead to nonplanar interfaces or interdiffusion. In heteroepitaxial growth misfit between the bulk lattice parameters of the substrate and the adlayer introduces strain in the interface, and pseudomorphic layers with misfit dislocations may form. The surface morphology of films depends on both the deposition temperature and the deposition rate, since the processes of nucleation and surface diffusion depend strongly on the temperature and crystallographic orientation of the surface. The growth of smooth epitaxial layers usually takes place in the step flow mode at high temperatures, whereas 3D islands with a distribution of sizes may form at lower temperatures [2-4]. Two different types of 3D islands can be formed in the SK mode. Initially, small regularly shaped dislocation-free islands are formed which are called hut clusters. At higher coverages larger islands form and strain relief occurs so the lattice parameter of the adlayer is closer to its bulk value.
It is well established that the growth of $\mathrm{Ge}$ on $\mathrm{Si}(001)$ progresses according to the following steps. For small coverages up to 3 monolayer (ML) epitaxial growth occurs - the layers are continuous and the surface has a $2 \times$ $N$ reconstruction (typically with $N=8-12$ ) with missing dimer rows which allow some strain relaxation. As the growth continues the missing dimers are partially filled, and if the temperature and deposition rate are chosen correctly then nanoscale hut clusters start to form. Eventually after depositing the amount of Ge equivalent to a coverage of about $6 \mathrm{ML}$ most of the substrate surface is covered with hut clusters. At higher coverages sometimes larger Ge islands are formed as described in Ref. [5]. It is important to note that the nanoscale clusters are metastable and are formed only at growth temperatures below $530{ }^{\circ} \mathrm{C}$.

The name "hut cluster" was introduced by Mo et al. [6] in the course of their scanning tunneling microscope (STM) investigations on the epitaxial growth of Ge on $\mathrm{Si}(001)$. The islands look like elongated huts all of similar width, typically about $150 \AA$ and with variable length depending on the deposition conditions. The external surfaces are bounded by $\{105\}$ facets, and at the interface the hut clusters are in registry with the $\mathrm{Si}(001)$ substrate; thus they are parallel to the $\langle 100\rangle$ or the $\langle 010\rangle$ directions. Two important characteristics of the hut clusters are the lack of dislocations at the interface and an elastic deformation which partially relaxes the strain associated with the $4.2 \%$ misfit between the lattice parameters of $\mathrm{Ge}$ and $\mathrm{Si}$ [7].

The importance of hut clusters for the relaxation of strain has been studied by several authors [8-10]. Williams et al. [11] performed x-ray diffraction measurements of the lattice parameters parallel to the substrate surface and were able to distinguish a broad peak originating from $\mathrm{Ge}$ scattering from the sharp Si signal, and they concluded that 
the onset of strain relaxation coincided with the appearance of clusters. However, it is not clear to what extent the scattering they observed originated from the hut clusters.

Scanning probe microscopy techniques have revealed a vast amount of information concerning thin film growth and the shapes and size distributions of such islands [6,1214]. However, it is difficult to determine the internal atomic structure of the islands, the strain fields, and the interface structure of small islands. We report here what we believe to be the first determination of the internal structure of hut clusters by combined STM and x-ray diffraction measurements. The strain distribution of small nanocrystals on a substrate, here $\mathrm{Ge}$ islands on $\mathrm{Si}(001)$, has been determined separately from the strain in the substrate and in the layers between the islands. We find that the $\mathrm{Ge}$ layers are almost fully strained near the interface but relax progressively towards the apex of the hut clusters.

The samples were prepared in a UHV system with STM, reflection high energy electron diffraction (RHEED), low energy electron diffraction (LEED), and controlled deposition facilities. The substrates were polished $\mathrm{Si}(001)$ wafers, cleaned by prolonged outgassing at $875 \mathrm{~K}$ followed by flashing to $1425 \mathrm{~K}$. STM measurements revealed clean well-ordered surfaces, and the step distribution indicated that the miscut angle of the wafers was less than $0.2^{\circ}$. Germanium was evaporated from an effusion cell at a deposition rate of about $0.6 \mathrm{ML} / \mathrm{min}$. Different substrate temperatures were tried to optimize the growth of the hut clusters and the best results were obtained with a substrate temperature of $430{ }^{\circ} \mathrm{C}$. After deposition and allowing to cool to room temperature the sample was inspected with LEED and STM for the presence of hut clusters and the absence of macroclusters which have characteristic $\{113\}$ facets. In the LEED pattern the hut clusters give rise to extra reflections in the [100] bulk direction [15]. Figure 1 shows a representative STM image from a sample prepared by depositing the equivalent of $6 \mathrm{ML} \mathrm{Ge}$ at a substrate temperature of $430{ }^{\circ} \mathrm{C}$. The average height of the hut clusters is only $13 \AA$. After preparation and characterization with electron diffraction and STM the samples were transferred under ultrahigh vacuum to a small chamber with a hemi-

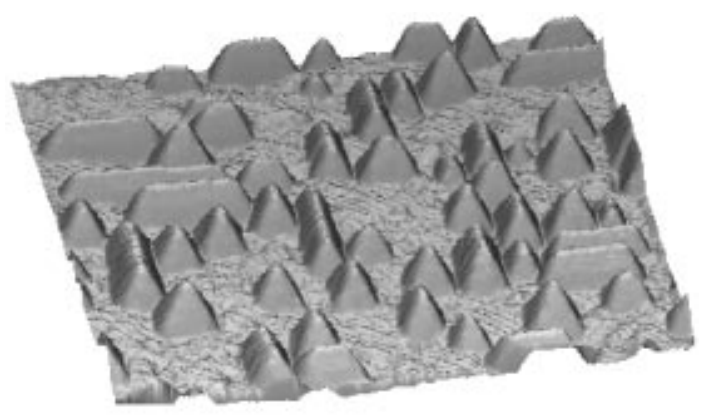

FIG. 1. STM image with dimensions of $2200 \times 2200 \AA$ of a sample prepared by depositing $6 \mathrm{ML} \mathrm{Ge}$ on $\mathrm{Si}(001)$ at a temperature of $430{ }^{\circ} \mathrm{C}$. spherical Be window, which was mounted on the diffractometer for the $\mathrm{x}$-ray measurements with synchrotron radiation.

The x-ray measurements were performed on the vertical scattering diffractometer at the BW2 wiggler beam line in HASYLAB (DESY Hamburg). The photon energy was $9.4 \mathrm{keV}$ and slits limited the width of incident and scattered beams to 1 and $1.5 \mathrm{~mm}$, respectively, thus defining the effective diffracting area on the sample. The angle of grazing incidence was kept constant and equal to the critical angle of total external reflection for $\mathrm{Si}\left(0.2^{\circ}\right)$. We use the coordinate notation of the bulk Si crystal with the (001) direction perpendicular to the sample surface. Scans were performed parallel to the surface by varying either $h$ or $k$, keeping the momentum transfer perpendicular to the surface $l$ constant. An extensive data set including several symmetry equivalent reflections was measured.

Figure 2 shows some representative examples of $h$ scans through the crystal truncation rods (CTR) which run through the (111), (202), and (400) bulk Bragg points (the experimental data points are indicated by open circles). In addition to the central peak, side peaks are observed which run along $\{105\}$ directions and have an asymmetrical intensity distribution around the central peak. These side peaks originate from the $\{105\}$ facets of the hut clusters. As will be shown below the strain distribution in the hut clusters can be deduced from the analysis of these profiles.

The central peak in the measured scans is due to the crystal truncation rod arising from all of the (001) interfaces and includes contributions from the surface of the Ge layers between the huts and both the buried interfaces beneath the huts and below the Ge layer in the area between the huts. The STM images show that the $2 \times N$ reconstructed Ge layers between the hut clusters is rather rough. There will be interference effects between the multiple contributions from the various (001) interfaces which depend on the detailed atomic geometry. Hence, the central peak cannot be modeled readily and is excluded from the data analysis. On the other hand, the intensities of the rods in the $\langle 105\rangle$ directions are due solely to the hut clusters and by measuring them we can distinguish uniquely the scattering from the atoms in the hut clusters from all other contributions. It should be noted that despite the grazingincidence geometry the dimensions of the hut clusters are small compared to the penetration depth of the $\mathrm{x}$ rays and the extinction length so it is permissible to apply kinematic scattering theory.

In the model calculations we included contributions from all of the atoms in the hut clusters to determine the diffracted intensity. As a starting point we assume that the hut cluster is laterally completely strained and thus all the Ge atoms in the hut clusters are at in-plane Si lattice sites. This model predicts the $\{105\}$ rods, but the asymmetric intensity distribution is not reproduced as shown in Fig. 3(a). To account for the $4.2 \%$ misfit 

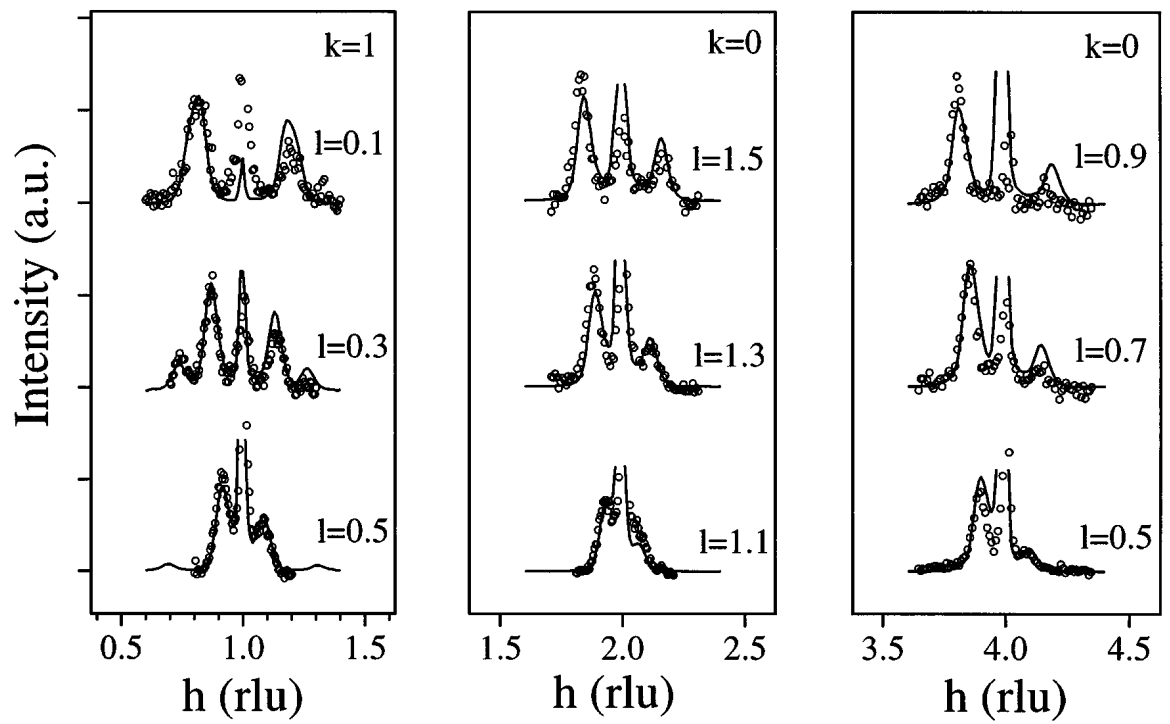

FIG. 2. Calculated and measured x-ray diffraction profiles around $\left(\begin{array}{lll}1 & 1 & l\end{array}\right),\left(\begin{array}{lll}2 & 0 & l\end{array}\right)$, and $\left(\begin{array}{llll}4 & 0 & l\end{array}\right)$. The circles represent the experimental data points, and the solid lines are the calculated profiles using the model described in the text which includes the inhomogeneous strain field in the hut cluster.

between the Ge and $\mathrm{Si}$ lattices we have to introduce strain relaxation. As a first approximation we assume that the clusters are long and narrow and set the lattice parameter in the direction along the long side of the hut cluster to be equal to the Si lattice constant. Along the short side we allow the in-plane Ge lattice parameter $a_{y}(z)$ to expand laterally with a power-law dependence as a function of height $z$ above the interface. We have tried different functional forms and have found that a quadratic dependence of $a_{y}(z)$ gives the best fit to the experimental data. The height variation of the lattice constant is given by

$$
a_{y}(z)=a_{\text {bottom }}+\left(a_{\text {top }}-a_{\text {bottom }}\right)\left(\frac{z}{h}\right)^{2},
$$

where $h$ is the height of the hut cluster, $a_{\text {top }}$ and $a_{\text {bottom }}$ are the lateral lattice parameters at the top and bottom of the cluster. One expects $a_{\text {bottom }}$ to be close to the $\mathrm{Si}$ lattice parameter and that $a_{\text {top }}$ will tend towards the lattice parameter of bulk Ge. The vertical lattice parameters are calculated using the Poisson ratio $(\nu=0.277)$. As indicated schematically in Fig. 3 this strain profile allows the clusters to relax from a maximum strain near the substrate to a minimum at the apex of the cluster. The lower part of Fig. 3 indicates the effect of strain on the calculated structure factors, and it can be seen that the inhomogeneous strain produces an asymmetrical intensity distribution. The effects are particularly pronounced for large momentum transfer, so we have measured out as far as $\left(\begin{array}{lll}5 & 0 l\end{array}\right)$. Although the model given above to describe the strain profile is clear and plausible, it certainly represents an oversimplification since it neglects a number of aspects. The strain field in the $\mathrm{Si}$ substrate, the bowing distortion of the Ge lattice planes, and the reconstruction of the sur- faces were not included in the model; such considerations go beyond the scope of the present investigation [16].

The size distribution of the hut clusters is an essential input parameter for the model calculations. From the STM images of the samples investigated by x-ray diffraction the average length of the clusters was determined to be $300 \AA$ and the average width $130 \AA$ with a surface coverage of $70 \%$. By combining the information from STM and x-ray diffraction a satisfactory fit to the measured data could be obtained by varying only the in-plane lattice parameters $a_{\text {bottom }}, a_{\text {top }}$, and a scale factor. Figure 2 shows the comparison between the experimental and calculated

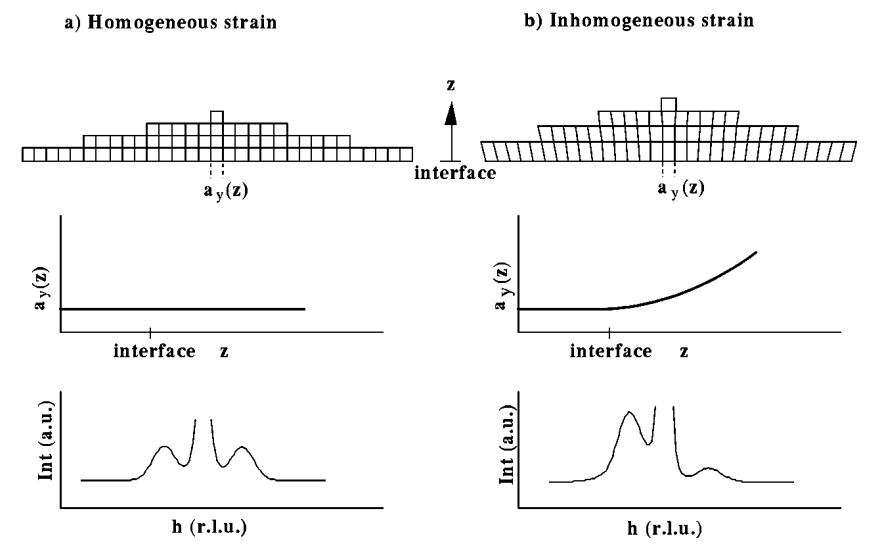

FIG. 3. Schematic diagram illustrating the models of the strained hut clusters. The upper part shows cross sections of clusters with and without inhomogeneous strain; the middle section shows the variation of the lattice parameter $a_{y}(z)$ as function of the height $z$ above the interface. At the bottom the corresponding calculated structure factors for $h$ scans through the $\left(\begin{array}{lll}4 & 0 & 0.5\end{array}\right)$ reciprocal lattice point are shown. 
$h$ scans. The open circles indicate the measured profiles and the solid lines the profiles calculated using the strain field in the hut cluster given by the fit procedure. From the model calculations we find the onset relaxation at the bottom of the hut cluster is $0.5 \%$ and the maximum strain relaxation at the apex of the cluster is $4.2 \%$. This means that the strain is relaxed uniformly through the hut cluster and that no dislocations are formed.

In conclusion, we have determined the strain field inside small Ge hut clusters on $\mathrm{Si}(001)$ by performing model calculations using kinematic theory to simulate the measured x-ray diffraction profiles. We find that the strain can be described by a continuous relaxation from the almost fully strained $\mathrm{Ge}-\mathrm{Si}$ interface towards the unstrained $\mathrm{Ge}$ atoms at the apex of the hut clusters. We have shown that STM combined with synchrotron $\mathrm{x}$-ray diffraction is a sensitive technique for determining the nonuniform strain and that it is possible to distinguish uniquely the scattering from the hut cluster atoms from all other contributions.

[1] See, for example, A. Zangwill, Physics at Surfaces (Cambridge University Press, Cambridge, 1988).

[2] J. E. Van Norstrand, S. J. Chey, M.-A. Hasan, D. G. Cahill, and J. E. Greene, Phys. Rev. Lett. 74, 1127 (1995).

[3] J. A. Stroscio, D. T. Pierce, M. D. Stiles, A. Zangwill, and L. M. Sander, Phys. Rev. Lett. 75, 4246 (1995).
[4] J. Tersoff, A. W. Renier van der Gon, and R. M. Tromp, Phys. Rev. Lett. 72, 266 (1994).

[5] M. Tomitori, K. Watanabe, M. Kobayashi, and O. Nishikawa, Appl. Surf. Sci. 76/77, 322 (1994).

[6] Y.-W. Mo, D. E. Savage, B. S. Swartzentruber, and M. G. Lagally, Phys. Rev. Lett. 65, 1020 (1990).

[7] D. J. Eaglesham and M. Cerullo, Phys. Rev. Lett. 64, 1943 (1990).

[8] J. Tersoff and R. M. Tromp, Phys. Rev. Lett. 70, 2782 (1993).

[9] P. Zeppenfeld, M. Krzyzowski, C. Romainczyk, G. Comsa, and M. G. Lagally, Phys. Rev. Lett. 72, 2737 (1994).

[10] V.A. Shchukin, N. N. Ledentsov, P.S. Ko'ev, and D. Bimberg, Phys. Rev. Lett. 75, 2968 (1995).

[11] A. A. Williams, J. M. C. Thornton, J. E. MacDonald, R. G. van Silfhout, J.F. van der Veen, M.S. Finney, A. D. Johnson, and C. Norris, Phys. Rev. B 43, 5001 (1991).

[12] Z. Gai, H. Ji, Y. He, C. Hu, R. G. Zhao, and W. S. Yang, Surf. Sci. 338, L851 (1995).

[13] J. R. Heffelfinger, M. W. Bench, and C. B. Carter, Surf. Sci. 343, L1161 (1995).

[14] C. W. Snyder, B. G. Orr, D. Kessler, and L. M. Sander, Phys. Rev. Lett. 66, 3032 (1991).

[15] C. E. Aumann, Y.-W. Mo, and M. G. Lagally, Appl. Phys. Lett. 59, 1061 (1991).

[16] J. Tersoff, C. Teichert, and M. G. Lagally, Phys. Rev. Lett. 76, 1675 (1996). 